\title{
Efficacy and safety of ledipasvir/sofosbuvir in 5,028 Mongolian patients infected with genotype 1 hepatitis $C$ virus: A multicenter study
}

Oidov Baatarkhuu ${ }^{1,2,3,4, *}$, Jae Seung Lee ${ }^{2, *}$, Jazag Amarsanaa ${ }^{4,5, *}$, Do Young Kim², Sang Hoon Ahn², Nyamsuren Naranzul ${ }^{1,4}$, Damba Enkhtuya ${ }^{4,5}$, Nagir Choijamts ${ }^{4,5}$, Purev Batbayar ${ }^{4,5}$, Radnaa Otgonbayar ${ }^{6}$, Bat-Ulzii Saruul ${ }^{4,7}$, Chuluunbaatar Gantuul ${ }^{4,7}$, Baljinnyam Gegeebadrakh ${ }^{4,8}$, Narangerel Tuvshinbayar ${ }^{4,8}$, Dorjgotov Badamsuren ${ }^{4,9}$, Galsan Ulzmaa ${ }^{4,9}$, Jamiyandorj Otgonbold ${ }^{10}$, and Kwang-Hyub Han ${ }^{2}$

'Department of Infectious Diseases, School of Medicine, Mongolian National University of Medical Sciences, Ulaanbaatar, Mongolia; 2Department of Internal Medicine, Yonsei University College of Medicine, Seoul, Korea; ${ }^{3}$ Mongolian Academy of Medical Sciences, Ulaanbaatar; ${ }^{4}$ Mongolian Association for the Study of Liver Diseases; ${ }^{5}$ Department of Hepatology, Happy Veritas Liver Diagnostics Center, Ulaanbaatar; ${ }^{6}$ Department of Internal Medicine, University General Hospital, Mongolian National University of Medical Sciences, Ulaanbaatar; ${ }^{7}$ Department of Hepatology, National Center for Communicable Diseases, Ulaanbaatar; ${ }^{8}$ Department of Gastroenterology, Second State Central Hospital, Ulaanbaatar; ${ }^{9}$ Department of Gastroenterology, Third State Central Hospital, Ulaanbaatar;

${ }^{10}$ School of Dentistry, Mongolian National University of Medical Sciences, Ulaanbaatar, Mongolia

Graphical Abstract

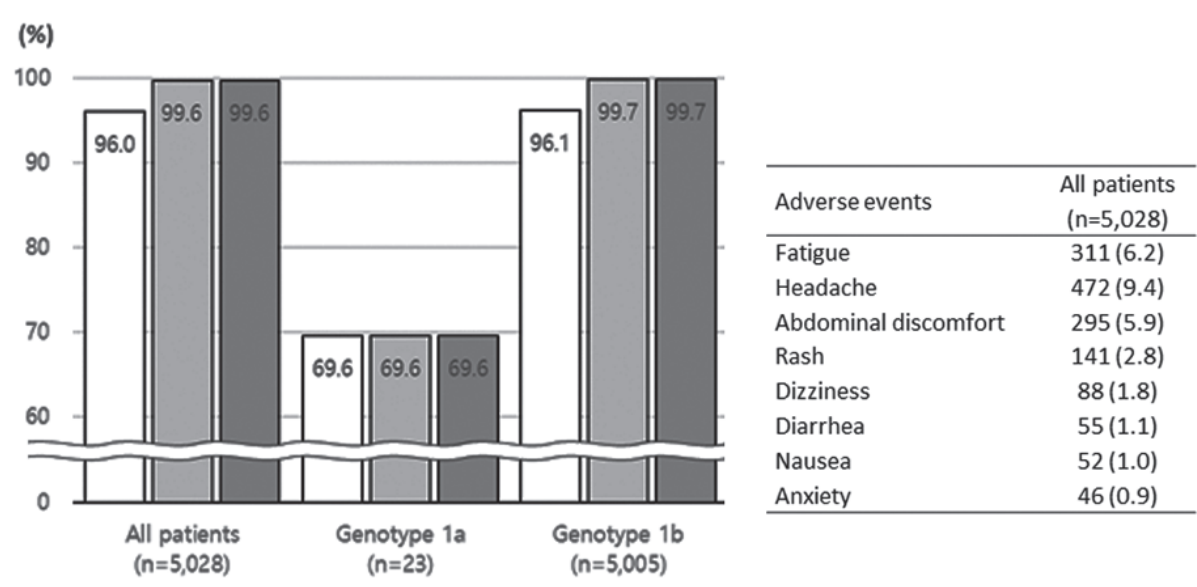

QRVR QETR QSVR12
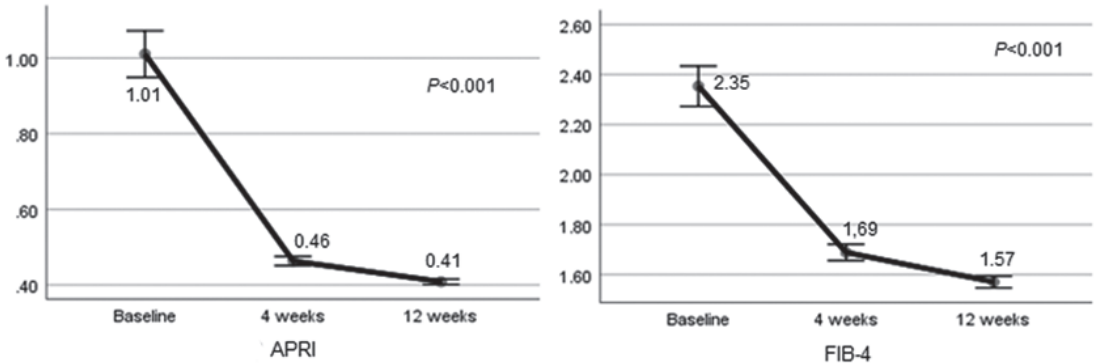

Ledipasvir/Sofosbuvir treatment in 5,028 Mongolian patients with genotype 1 hepatitis $C$ virus

- High efficacy (especially in genotype $1 b$ patients) and safety (without severe adverse events)

- Improvement of non-invasive fibrosis scores after Ledipasvir/Sofosbuvir treatment 
Background/Aims: Ledipasvir/sofosbuvir (LDV/SOF) shows high efficacy and safety in patients with genotype 1-hepatitis $\mathrm{C}$ virus (HCV). We aimed to investigate the efficacy and safety of LDV/SOF in real-world Mongolian patients.

Methods: Between 2015 to 2019, 23 (0.5\%) and 5,005 patients (99.5\%) with genotype 1a and 1b HCV, respectively, were treated with a fixed-dose tablet containing $90 \mathrm{mg}$ ledipasvir and $400 \mathrm{mg}$ sofosbuvir for 12 weeks, and 81 patients (1.6\%) with previous experience of interferon (IFN)-based treatment received additional 1,000 mg ribavirin. HCV RNA was measured at 4, 12, and 24 weeks after the first dose to determine rapid virologic response, end of treatment response (ETR), and sustained virologic response at 12 weeks after end of treatment (SVR12).

Results: Most patients ( $n=5,008 ; 99.6 \%)$ achieved ETR and SVR12 without virologic relapse. Patients with genotype 1a showed low rates of ETR and SVR12 in only 16 patients (69.6\%). There was no significant difference in SVR12 rate between patients regardless of IFN experience $(n=81 ; 1.6 \%)$, cirrhosis $(n=1,151 ; 22.9 \%)$, HCV RNA $>6 \times 10^{6} \mathrm{IU} / \mathrm{mL}(\mathrm{n}=866 ; 17.2 \%)$, or liver stiffness $>9.6 \mathrm{kPa}(\mathrm{n}=1,721 ; 34.2 \%)(100.0 \%, 99.3 \%, 99.4 \%$, and 99.4\%, respectively). No severe adverse events (AEs) were reported, and there was no dose reduction or interruption due to AE. The most common AEs were headache $(n=472$; $9.4 \%)$, fatigue $(n=306 ; 6.2 \%)$, abdominal discomfort $(n=295 ; 5.9 \%)$, and skin rash $(n=141 ; 2.8 \%)$.

Conclusions: LDV/SOF showed high efficacy and safety for patients with genotype 1, especially $1 \mathrm{~b} \mathrm{HCV}$, in Mongolia. The real-world data might be applicable to patients in other Asian-Pacific countries. (Clin Mol Hepatol 2021;27:125-135)

Keywords: Hepatitis C, Chronic; Ledipasvir; Sofosbuvir; Mongolia; Real-world

\section{Study Highlights}

We investigated the high efficacy and safety of ledipasvir/sofosbuvir regimen in real-world Mongolian patients with genotype 1 hepatitis C virus, especially in patients with genotype $1 \mathrm{~b}$. Although LDV/SOF treatment is not a novel issue, this large-scale cohort study was aimed to show the efficacy and safety of an affordable treatment for developing countries such as Mongolia. This study could be a good example of successful hepatitis $C$ treatment practice in developing countries where healthcare budgets are limited.

\section{INTRODUCTION}

The prevalence of hepatitis C virus (HCV) infection in Mongolia is estimated to be $15.6 \%$ based on a national seroepidemiologic data. ${ }^{1-3}$ Such a high rate of HCV infection in Mongolia might be due to reuse of needles before 1990, traditional medicine including bloodletting, and other unsafe medical or dental procedures. Interestingly, the most common HCV genotype in Mongolia was found to be $1 b$, accounting for $98 \%$ of all genotypes. ${ }^{2}$

since the introduction of direct-acting antivirals (DAAs) includ-

\begin{abstract}
Abbreviations:
AEs, adverse events; ALT, alanine aminotransferase; APRI, aspartate aminotransferase to platelet ratio index; AST, aspartate aminotransferase; CBC, complete blood count; $\mathrm{Cl}$, confidence interval; DAAs, direct-acting antivirals; EOT, end of treatment; ETR, end of treatment response; FIB-4, fibrosis-4; HCV, hepatitis C virus; HIV, human immunodeficiency virus; IQR, interquartile range; LDV/SOF, ledipasvir/sofosbuvir; LS, liver stiffness; LSM, liver stiffness measurement; OR, odds ratio; PEG-IFN, pegylated interferon; RASs, resistanceassociated substitutions; ROC, receiver operating characteristic; RVR, rapid virologic response; SVR, sustained virologic response
\end{abstract}

\section{Corresponding author : Do Young Kim}

Department of Internal Medicine, Yonsei University College of Medicine, 50-1 Yonsei-ro, Seodaemun-gu, Seoul 03722, Korea

Tel: +82-2-2228-1992, Fax: +82-2-393-6884

E-mail:dyk1025@yuhs.ac

https://orcid.org/0000-0002-8327-3439

\section{Sang Hoon Ahn}

Department of Internal Medicine, Yonsei University College of Medicine, 50-1 Yonsei-ro, Seodaemun-gu, Seoul 03722, Korea

Tel: +82-2-2228-1936, Fax: +82-2-393-6884

E-mail:ahnsh@yuhs.ac

https://orcid.org/0000-0002-3629-4624

*Three authors contributed equally to this article as co-first authors. 
ing boceprevir and telaprevir in 2011, the new DAA ledipasvir/sofosbuvir (LDV/SOF) in 2014 opened a new era of interferon-free, all-oral agents for HCV treatment. ${ }^{4}$ A fixed dose of ledipasvir, a NS5A inhibitor, and sofosbuvir, a nucleotide analogue NS5B polymerase inhibitor, showed a promising antiviral efficacy and safety in genotype $1 \mathrm{HCV}$-infected patients. In a phase 3 pivotal study, the overall sustained virologic response (SVR) rates were $99 \%$ in treatment-naïve patients who received 12 weeks of LDV/SOF. ${ }^{5}$ Moreover, in genotype $1 \mathrm{HCV}$ patients who had experienced prior interferon-based treatment, 12 weeks of LDV/SOF plus ribavirin resulted in $96 \%$ of SVR rates. ${ }^{6}$

In the era of interferon or pegylated interferon (PEG-IFN), Mongolian patients rarely underwent antiviral therapy because the cost was high and the access to medical resources was significantly limited. However, according to the approval of the implementation of the Hepatitis Prevention, Control and Elimination Program between 2016 and 2020 by the Mongolian government, treatment campaign for HCV started from January 2016. Fortunately, Gilead Sciences, Inc. has successfully implemented an access program, in which the cost of LDV/SOF was remarkably reduced to 300 US dollars per bottle. To date, more than 10,000 Mongolian patients infected with HCV have received brand LDV/ SOF (Harvoni ${ }^{\circledR}$; Gilead Sciences, Inc., Foster City, CA, USA).

There have been few real-life data regarding brand or generic LDV/SOF for HCV patients worldwide. ${ }^{7}$ However, the sample size was small. Thus, the aim of this study was to report the efficacy and safety of brand LDV/SOF in 5,028 Mongolian patients infected with genotype $1 \mathrm{HCV}$ who received LDV/SOF or LDV/SOF plus ribavirin for 12 weeks.

\section{PATIENTS AND METHODS}

\section{Patients eligibility}

In this retrospective cohort study, patients who met the following criteria were included: 1) patients aged 18-70 years; 2) patients with chronic HCV infection documented by anti-HCV or by patient's statement; 3) patients with HCV genotype 1a or 1b; 4) treatment-experienced or -naive patients; 5) patients with negative results for hepatitis $A$ and $B$ as well as human immunodeficiency virus (HIV); 6) patients without decompensation of liver function, such as ascites and hepatic encephalopathy; 7) patients without diagnosis of hepatocellular carcinoma at enrollment; 8) patients with normal value of baseline serum alpha-fetoprotein;
9) patients with normal range of baseline serum creatinine; 10) patients who have completed follow-up for 12 weeks after the end of treatment (EOT); and 11) patients who were treated with only brand LDV/SOF.

\section{Laboratory tests and data collection}

All included patients were subjected to laboratory tests at the beginning of treatment, including complete blood count (CBC), alanine aminotransferase (ALT), aspartate aminotransferase (AST), total bilirubin, serum albumin, prothrombin time, IgG anti-hepatitis A virus, hepatitis B virus surface antigen, anti-HIV, and antiHCV. Anti-HCV reactivity was assayed using a HISCL 5000 analyzer (Sysmex Corp., Kobe, Japan), and serum HCV RNA was quantitated using a Roche $\operatorname{COBAS}^{\circledR}$ TagMan HCV Test (V3.0, cutoff value, $15 \mathrm{IU} / \mathrm{mL}$; Roche Molecular Systems, Branchburg, NJ, USA). HCV genotyping was performed using a gene sequencing assay.

Laboratory tests, including CBC, ALT, AST, total bilirubin, serum albumin, prothrombin time, and serum HCV RNA quantitation, were repeated at 4 weeks, EOT, and 12 weeks after EOT.

Approximately 65,000 patients infected with HCV received LDV/SOF between 2016 and 2020 by treatment campaign in Mongolia

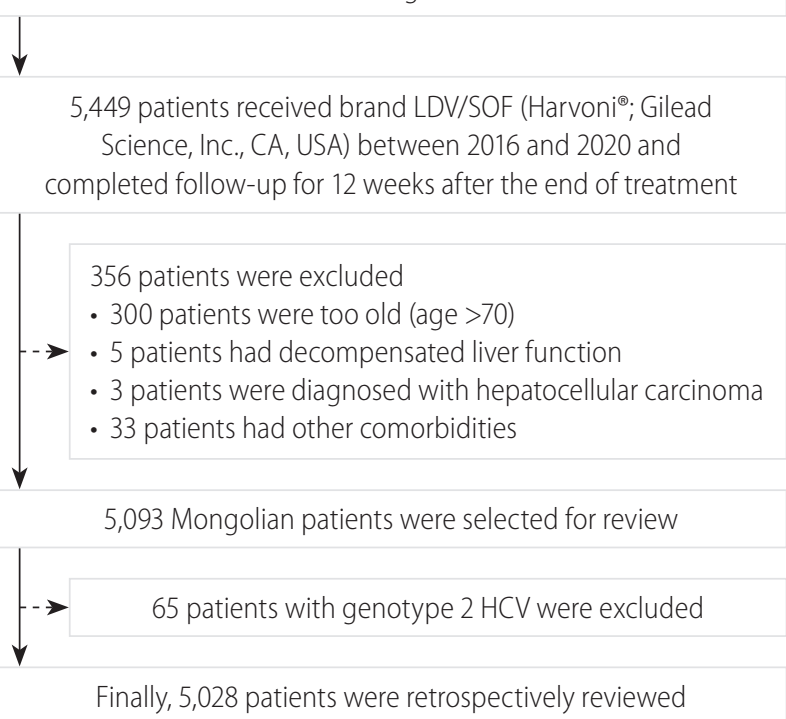

Figure 1. Flowchart of patient enrollment. HCV, hepatitis C virus; LDV/ SOF, ledipasvir/sofosbuvir. 


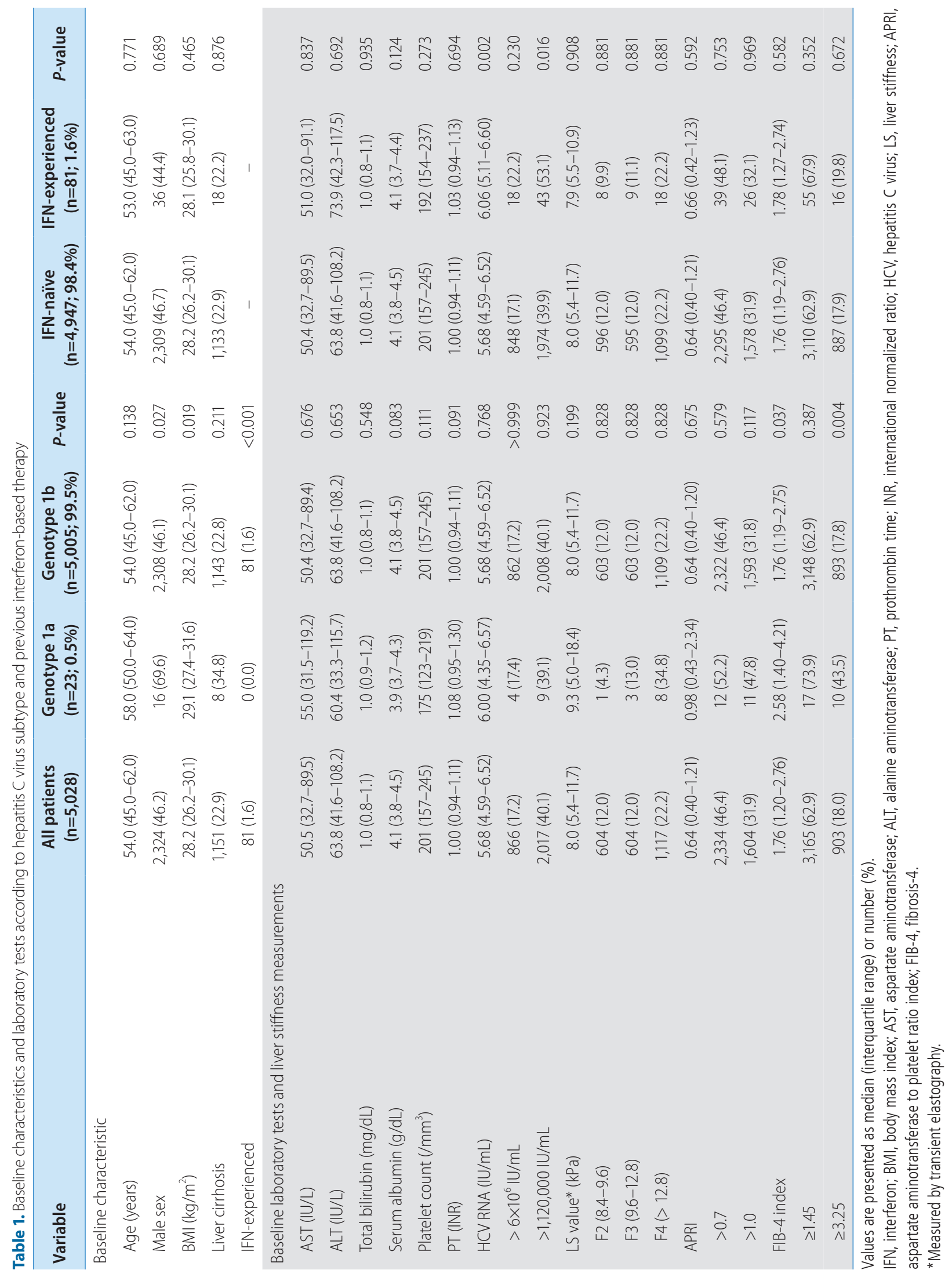




\section{Ultrasonographic evaluation and liver stiffness measurement}

Before administration of LDV/SOF, baseline ultrasonographic examination and liver stiffness measurement (LSM) using transient elastography (FibroScan ${ }^{\circledR}$, Echosens, Paris, France) were conducted. LSM was performed by a single experienced technician who was blinded to the patient information. The results were expressed as kilopascals $(\mathrm{kPa})$, and the interquartile range (IQR) was defined as an index of intrinsic variability of LSM corresponding to the interval of LSM results containing $50 \%$ of the valid measurements between the 25th and 75th percentiles. The median value was considered representative of the elastic modulus of the liver. Only procedures with at least 10 valid measurements, a success rate of at least $60 \%$, and an IQR to median value ratio of $30 \%$ were considered reliable. ${ }^{8-10}$ The cutoff value was considered as 8.4 kPa for F2, 9.6 kPa for F3, and 12.8 kPa for F4 fibrosis according to a recent study." Because patients were subjected to LSM at baseline with active inflammation, overassessment of LSM could occur. Therefore, in this study, nodular liver surface combined with blunted edge in ultrasonographic evaluation and high liver stiffness (LS) value (>12.5 kPa) was considered as a relative definition of liver cirrhosis. ${ }^{12}$

Because repeated transient elastography was not feasible in Mongolia, AST to platelet ratio index (APRl; APRI = AST / 41 [as the upper limit of normal AST] / platelet count) and fibrosis-4 $\left(\right.$ FIB-4) index (FIB-4 index = age $\times$ AST / [platelet count $\times$ ALT $\left.\left.^{1 / 2}\right]\right)$, which are simple non-invasive fibrosis scoring systems, were calculated at each time point during treatment. Cutoff of significant and severe fibrosis were considered as 0.7 and 1.0 in APRI, and 1.45 and 3.25 in FIB-4, respectively. 11,13

\section{Antiviral therapy}

Patients with genotype $1 \mathrm{a}$ or $1 \mathrm{~b}$ received a fixed-dose combination tablet containing $90 \mathrm{mg}$ ledipasvir and $400 \mathrm{mg}$ sofosbuvir for 12 weeks, as only 12-week regimen was approved in Mongolia. If patients had a prior experience of PEG-IFN treatment for HCV, they received an additional fixed dose of 1,000 $\mathrm{mg}$ ribavirin during LDV/SOF treatment. ${ }^{5,6,14}$

\section{Assessment of antiviral efficacy and safety}

The primary efficacy endpoint was SVR, defined as undetectable HCV RNA at 12 weeks after EOT (SVR12). Rapid virologic response (RVR) and EOT response (ETR) were defined as completely undetectable HCV RNA at 4 weeks and EOT, respectively. The virologic relapse was defined as a detectable HCV RNA level after EOT in a patient who had undetectable HCV RNA during treatment. Non-response was defined as a detectable HCV RNA at EOT in a patient who did not experience virologic breakthrough. Any adverse events (AEs) that developed during antiviral therapy were recorded.

\section{Statistical analysis}

Continuous variables were expressed as median and IQR. Categorical variables were presented by frequency and percentage.

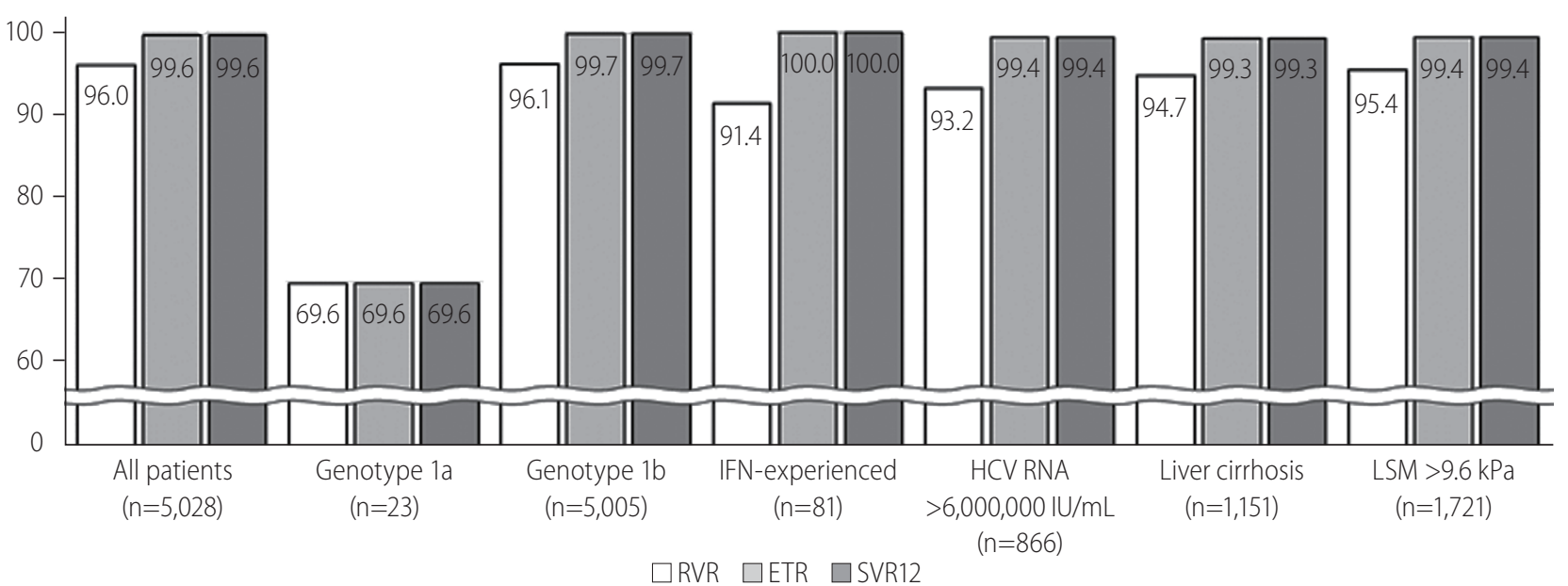

Figure 2. Rates of RVR, ETR, and SVR12. IFN, interferon; HCV, hepatitis C virus; LSM, liver stiffness measurement; RVR, rapid virological response; ETR, early virological response; SVR12, ustained virological response after 12 weeks of treatment. 


\section{CLINCAL and MOLECULAR}

Volume_27 Number_1 January 2021
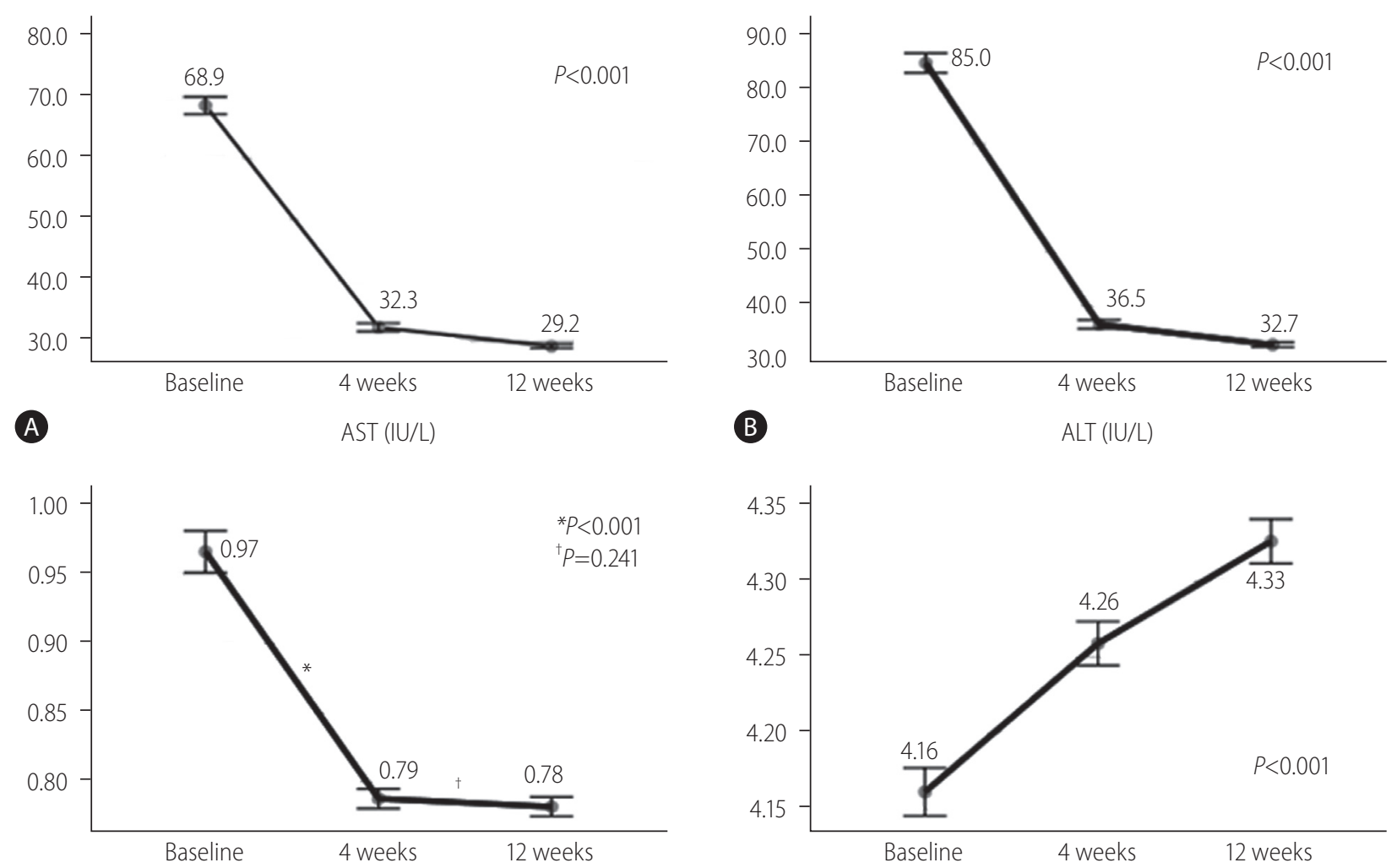

B

ALT (IU/L)
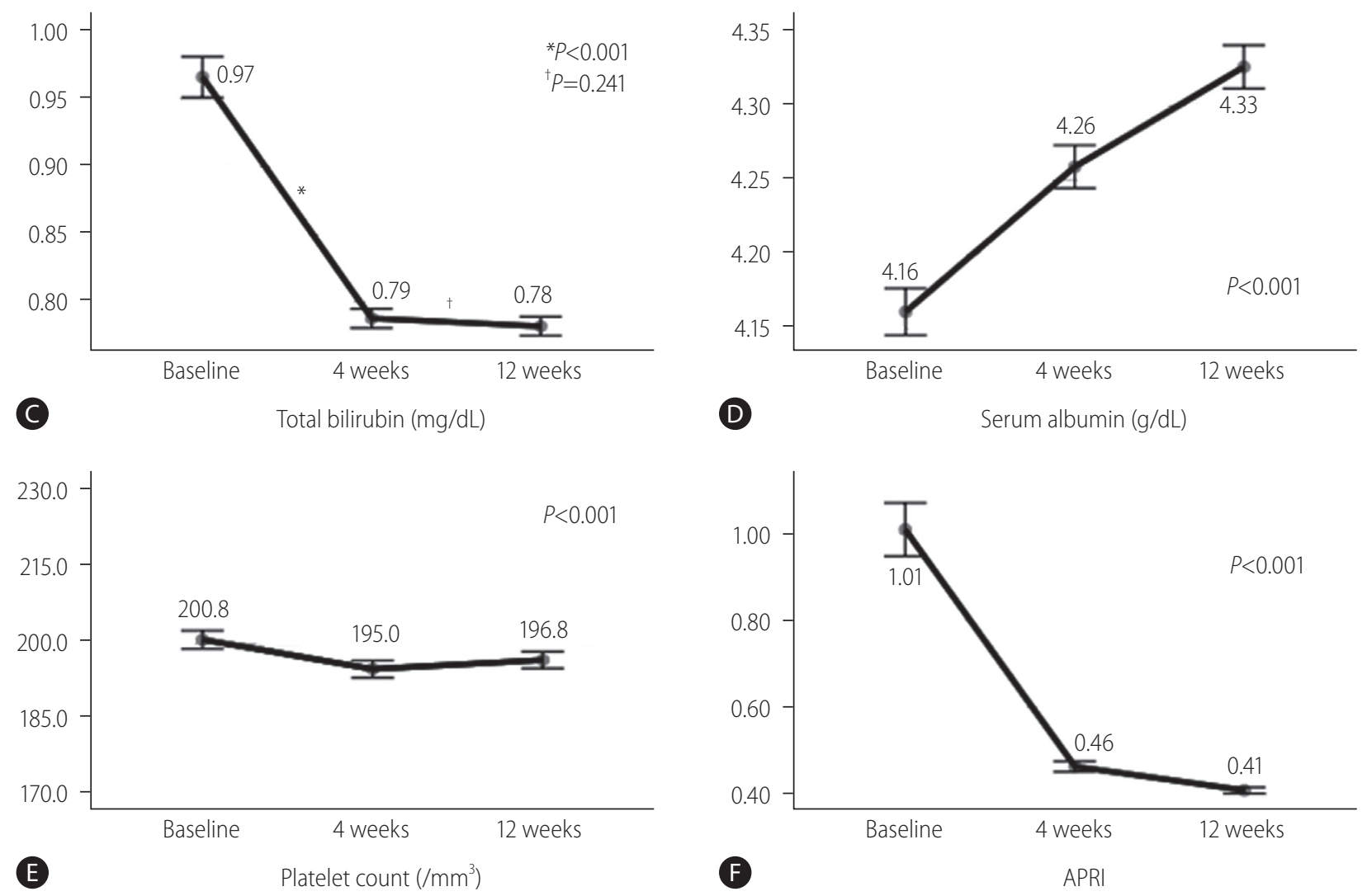

F

APRI

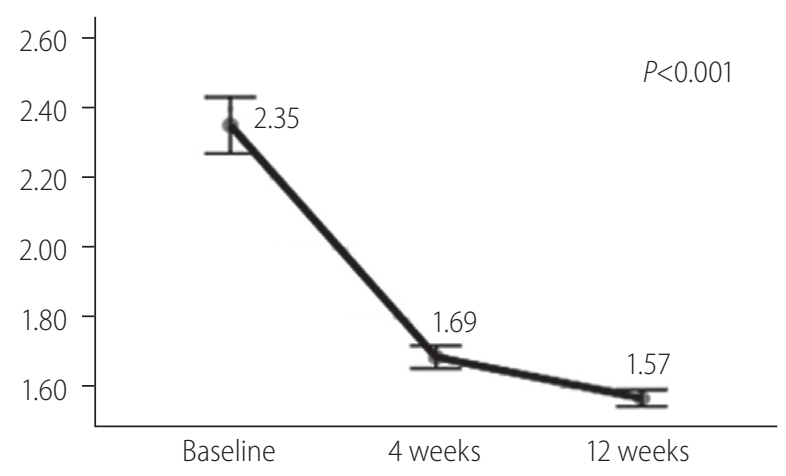

G FIB-4

Figure 3. Estimated marginal mean changes in laboratory test results and non-invasive fibrosis index after ledipasvir/sofosbuvir treatment: (A) aspartate aminotransferase (AST), (B) alanine aminotransferase (ALT), (C) total bilirubin, (D) serum albumin, (E) platelet count, (F) AST to platelet ratio index (APRI), and (G) fibrosis-4 index (FIB-4). ${ }^{*} P<0.001{ }^{\dagger} P=0.241$. 
Differences between continuous and categorical variables were examined for statistical significance using Student's $t$-test (or the Mann-Whitney test, as appropriate) and the chi-squared test (or Fisher's exact test, as appropriate). A generalized linear model using repeated measured analysis of variance (ANOVA) was used to assess the longitudinal changes in variables with a Bonferroni correction for multiple comparisons. Multivariate analysis was performed with adjustments for significant variables with $P<0.05$ in the univariate analyses using logistic regression model. Continuous variables were categorized using cutoffs that were clinically used or obtained with reference to the receiver operating characteristic (ROC) curve, and entered into the univariate and multivariate analyses. The odds ratio (OR) for SVR12 and its 95\% confidence interval $(\mathrm{Cl})$ were calculated for each selected variable. All statistical analyses were performed using the Statistical Package for Social Sciences (SPSS version 25.0; IBM, Armonk, NY, USA). A $P$ value $<0.05$ was considered statistically significant.

\section{RESULTS}

\section{Baseline characteristics and on-treatment laboratory tests}

According to the Hepatitis Prevention, Control and Elimination Program of the Mongolian government from January 2016, there were more than 10,000 patients treated with brand LDV/SOF for genotype 1 HCV between December 2015 and June 2019, and 5,449 patients completed follow-up for 12 weeks after the EOT. After excluding 421 patients, 5,028 patients who met the inclusion criteria were retrospectively analyzed (Fig. 1).

Baseline characteristics and on-treatment laboratory tests of the 5,028 patients are described in Table 1 and Supplementary Table 1. The median age was 54.0 years $(I Q R, 45.0-62.0)$. The proportion of male patients was $46.2 \%(n=2,324)$. Liver cirrhosis was clinically diagnosed in 1,151 patients (22.9\%). Additional ribavirin was administered during LDV/SOF treatment in only 81 (1.6\%) genotype $1 \mathrm{~b}$ patients who had previous PEG-IFN experience. The median AST and ALT levels were slightly high (50.5 and $63.8 \mathrm{IU} / \mathrm{L}$ ) at baseline. The median baseline LS value was $8.0 \mathrm{kPa}$ (IQR, 5.411.7), and 1,721 patients (34.2\%) showed LS value $>9.6 \mathrm{kPa}$.

Almost all ( $n=5,005 ; 99.5 \%)$ patients had HCV genotype $1 \mathrm{~b}$. Between genotype $1 \mathrm{a}$ and $1 \mathrm{~b}$, there was no significant difference at baseline regardless of sex distribution $(69.6 \%$ vs. $46.1 \%$ of male sex, $P=0.027$ ), body mass index (median $29.1 \mathrm{vs.} 28.2 \mathrm{~kg} / \mathrm{m}^{2}$,
$P=0.019$ ), and FIB-4 index (median 2.58 vs. $1.76, P=0.037$ ). At 4 weeks, genotype 1a patients showed slightly low serum albumin level (median 4.0 vs. $4.2 \mathrm{~g} / \mathrm{dL}$ ) as well as high prevalence of APRI above $1.0(21.7 \%$ vs. $5.8 \%)$ and FIB-4 above $3.25(21.7 \%$ vs. $6.9 \%)$ (all $P<0.05)$.

PEG-IFN-experienced patients tended to have higher baseline HCV RNA (log value 6.06 vs. 5.68, $P=0.002$ ). However, other variables were not significantly different between patients with or without PEG-IFN experience.

\section{Treatment outcomes}

Of total 5,028 patients with HCV genotype 1 infection, 5,008 (99.6\%) had successful HCV disappearance after 12 weeks of antiviral therapy. RVR, ETR, and SVR12 were analyzed in 4,825 (96.0\%), 5,008 (99.6\%), and 5,008 patients (99.6\%). All patients who showed RVR achieved ETR later, and patients who achieved ETR finally reached SVR12 without virologic relapse. RVR, ETR, and SVR12 were achieved by $69.6 \%(n=16), 69.6 \%$, and $69.6 \%$ of 23 patients with genotype $1 \mathrm{a}$, respectively. In contrast, the rates of RVR, ETR, and SVR12 in 5,005 patients with genotype $1 \mathrm{~b}$ were $96.1 \%(n=4,809), 99.7 \%(n=4,992)$, and $99.7 \%$, respectively. Most patients showed ETR and RVR regardless of the presence of PEG-IFN experience, high baseline HCV RNA $\left(>6 \times 10^{6} \mathrm{IU} / \mathrm{mL}\right)$, liver cirrhosis or indicators of advanced fibrosis including high LS, APRI, and FIB-4 values (Fig. 2, Supplementary Table 2).

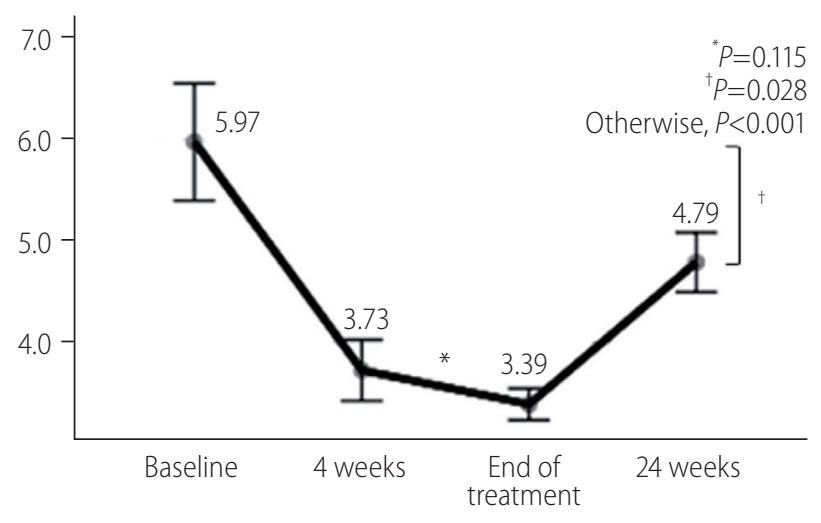

HCV RNA quanititation ( $\log I \mathrm{U} / \mathrm{mL})$

Figure 4. Estimated mean changes in log value of HCV RNA after treatment in 20 patients who failed to achieve end-treatment response and sustained virologic response. $\mathrm{HCV}$, hepatitis $C$ virus. ${ }^{*} P=0.115 .{ }^{\dagger} P=0.028$. 


\section{Laboratory result changes during antiviral therapy}

Changes in the estimated marginal mean of laboratory test results during antiviral therapy are described in Figure 3 and Supplementary Table 3. The estimated mean values of baseline AST and ALT levels were $68.9 \pm 0.7$ and $85.0 \pm 0.3 \mathrm{IU} / \mathrm{L}$, significantly decreased to $32.3 \pm 0.3$ and $36.5 \pm 0.4 \mathrm{IU} / \mathrm{L}$ at 4 weeks, and further decreased to $29.2 \pm 0.2$ and $32.7 \pm 0.2 \mathrm{IU} / \mathrm{L}$ at EOT, respectively (all $P<0.001)$. The estimated mean value of total bilirubin also decreased (0.96 to $0.78 \mathrm{mg} / \mathrm{dL}, P<0.001)$, and the mean serum albumin was slightly elevated $(4.2$ to $4.3 \mathrm{~g} / \mathrm{dL}, P<0.001)$ during antiviral therapy. Platelet count tended to slightly decrease, but almost similar $\left(200.8 / \mathrm{mm}^{3}\right.$ to $\left.196.8 / \mathrm{mm}^{3}, P<0.001\right)$. The estimated mean values of APRI and FIB-4 also reliably decreased (1.01 to 0.41 and 2.35 to 1.57 , respectively; all $P<0.001$ ).

\section{Virologic breakthrough after non-response}

Estimated mean HCV RNA quantitation in 20 patients (0.4\%) who showed non-response decreased until the EOT $(5.97 \pm 0.28$ to $3.39 \pm 0.08 \log \mathrm{IU} / \mathrm{mL}, P<0.001)$. However, at 12 weeks after the EOT, the log value of HCV RNA in non-responders was elevated to $4.79 \pm 0.14(P<0.001)$, which corresponded to virologic relapse after incomplete virologic response (Fig. 4, Supplementary Table 4).

Table 2. Multivariate analysis for complete end-treatment response and sustained virologic response at 12 weeks after treatment

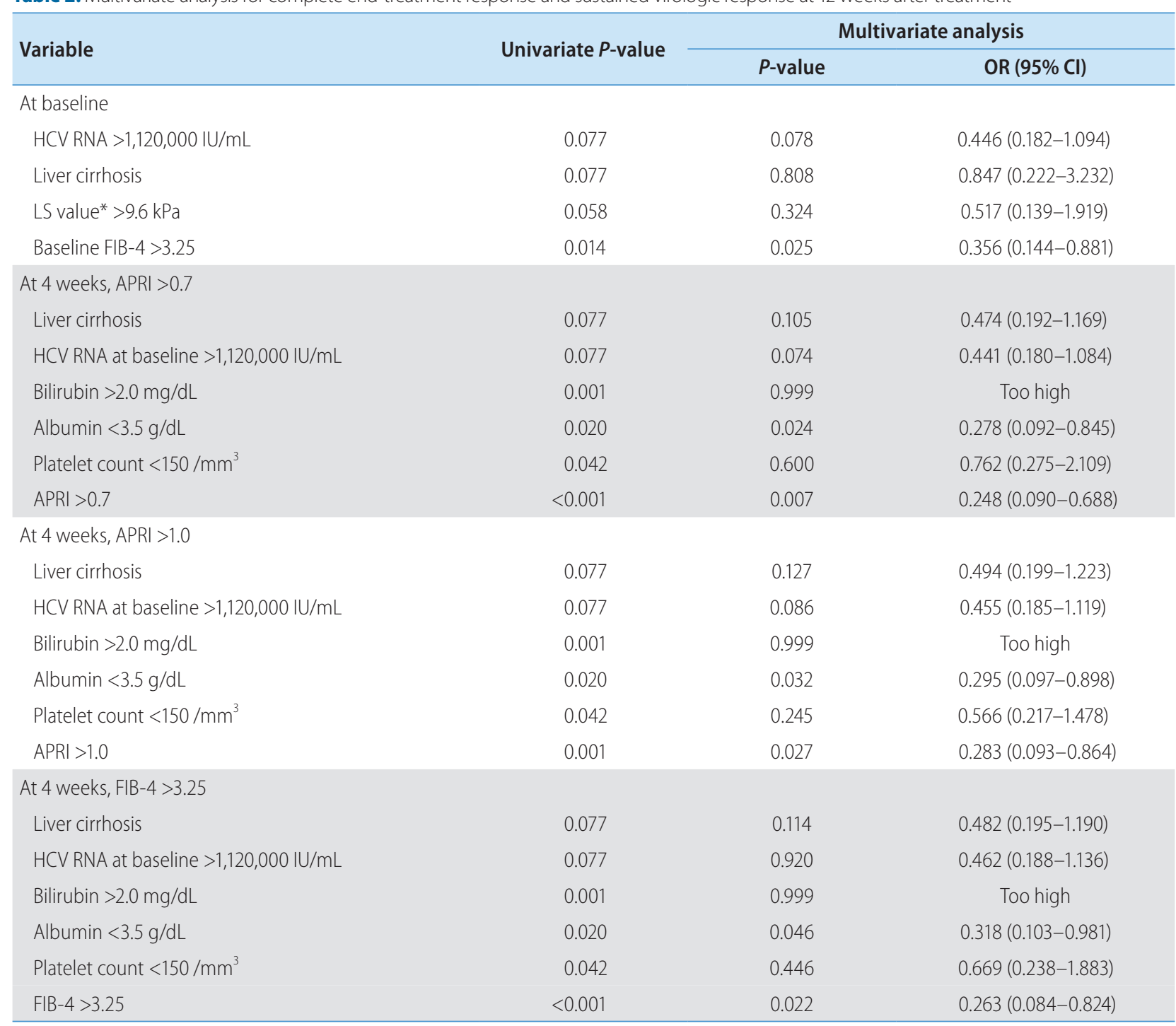

$\mathrm{OR}$, odds ratio; $\mathrm{Cl}$, confidence interval; $\mathrm{HCV}$, hepatitis C virus; LS, liver stiffness; FIB-4, fibrosis-4; APRI, aspartate aminotransferase to platelet ratio index.

*Measured by transient elastography. 


\section{Variables associated to ETR and SVR12}

In univariate analysis, variables at baseline, such as the presence of liver cirrhosis, high HCV RNA $\left(>1.12 \times 10^{6} \mathrm{IU} / \mathrm{mL}\right.$, cutoff by ROC curve), high LS (>9.6 kPa), and high FIB-4 (>3.25), and variables at 4 weeks, such as high bilirubin, low albumin, low platelet count, and APRI and FIB-4 above each cutoff, showed significant association with ETR and SVR12 achievement (all $P<0.05$ ) (Supplementary Table 5). Multivariate analyses at each time point based on these variables were subsequently performed (Table 2). At baseline, high FIB-4 (>3.25) (OR, 0.356; $P=0.025)$ showed reversed association with ETR and SVR12 achievement. At 4 weeks of treatment, non-invasive fibrosis scoring, such as APRI $>0.7$ (OR, 0.248), APRI >1.0 (OR, 0.283), and FIB-4 >3.25 (OR, 0.263) showed reversed association with ETR and SVR12 achievement, with serum albumin below $3.5 \mathrm{~g} / \mathrm{dL}$ (all $P<0.05$ ).

\section{AEs during antiviral therapy}

During 12 weeks of antiviral therapy, the most common AEs were headache, fatigue, abdominal discomfort, and skin rash, which occurred in 472 (9.4\%), 311 (6.2\%), 295 (5.9\%), and 141 patients (2.8\%). There was no difference in AE development between IFN-experienced and -naïve groups (Table 3).

\section{DISCUSSION}

In this real-world cohort study, 12-week treatment with LDV/ SOF ( \pm ribavirin) showed high effectiveness in 5,028 Mongolian patients infected with genotype $1 \mathrm{HCV}$, regardless of previous IFN experience. Compared with that in patients with genotype $1 \mathrm{~b}$ (99.6\%), SVR rate in genotype 1a was very low (69.6\%) compared with that in previous studies; however, the number is relatively small $(n=23 ; 0.5 \%)$. Non-responders $(n=20 ; 0.4 \%)$ also experienced reduction of viral load through 12 weeks of treatment with LDV/SOF; however, after the EOT, subsequent virologic breakthrough occurred in the 20 patients. No serious AE occurred during LDV/SOF treatment.

Similar to western guidelines, the recent Asian-Pacific guideline is based on the new era of DAAs on HCV treatment. ${ }^{15,16}$ Many DAAs are currently in use, and currently, pangenotypic DAAs such as sofosbuvir/velpatasvir and glecaprevir/pibrentasvir tend to be increasingly preferred. However, pangenotypic DAAs are not always affordable in some developing Asian-Pacific countries owing to relatively low healthcare budgets and resources. Nevertheless, LDV/SOF is a well-known powerful DAA for genotype $1 \mathrm{HCV}$ that showed $93 \%$ to $100 \%$ SVR rate. Recent European and Asian-Pacific guidelines still recommend 8-12 weeks of LDV/SOF for genotype $1 \mathrm{~b}$ and treatment-naïve genotype $1 \mathrm{a}, 4,5$, and $6 .{ }^{15,16}$ As this study was conducted in conjunction with a drug-producing company as part of a Mongolian healthcare program from 2016, approvement of the regimen was restricted to 12 weeks of LDV/SOF and ribavirin addition for treatment-experienced patients in Mongolia. $5,6,17,18$

To the best of our knowledge, this is the largest real-life study for genotype $1 \mathrm{HCV}$ treated with LDVISOF in a single country. The distribution of HCV genotypes was shown to be quite homogeneous in Mongolia in a previous study, which revealed that genotype $1 \mathrm{~b}$ accounted for $98.8 \%$ of all genotypes in Mongolia.' In this study, which was limited to genotype $1 \mathrm{HCV}, 99.5 \%$ of patients had genotype $1 \mathrm{~b}$ HCV. Such a high proportion of genotype

Table 3. Adverse events during antiviral therapy

\begin{tabular}{lcccc}
\hline Adverse event & All patients $(\mathbf{n}=\mathbf{5 , 0 2 8})$ & IFN-naïve $(\mathbf{n = 4 , 9 4 7 ; ~ 9 8 . 4 \% )}$ & IFN-experienced $(\mathbf{n}=\mathbf{8 1 ;} \mathbf{1 . 6 \% )}$ & $\boldsymbol{P}$-value \\
\hline Fatigue & $311(6.2)$ & $306(6.2)$ & $5(6.2)$ & $>0.999$ \\
Headache & $472(9.4)$ & $464(9.4)$ & $8(9.9)$ & 0.847 \\
\hline Abdominal discomfort & $295(5.9)$ & $290(5.9)$ & $5(6.2)$ & 0.811 \\
Rash & $141(2.8)$ & $138(2.8)$ & $3(3.7)$ & 0.496 \\
Dizziness & $88(1.8)$ & $86(1.7)$ & $2(2.5)$ & 0.653 \\
Diarrhea & $55(1.1)$ & $53(1.1)$ & $2(2.5)$ & 0.222 \\
Nausea & $52(1.0)$ & $51(1.0)$ & $1(1.2)$ & 0.572 \\
Anxiety & $46(0.9)$ & $46(0.9)$ & $0(0.0)$ & 1.000 \\
\hline
\end{tabular}

Values are presented as number (\%).

IFN, interferon. 
1b in Mongolian patients might be appropriate for LDV/SOF treatment, which showed an even higher overall SVR rate of $99.6 \%$ in genotype 1 than that in previous clinical trials. ${ }^{15,16}$

No virologic relapse was observed until 12 weeks after the EOT in this study. However, subsequent elevation of viral load was shown to be occur in 20 treatment-failure cases. A recent Japanese study suggested factors such as cirrhosis ( $O R, 4.19 ; P=0.014)$ and resistance-associated substitutions (RASs) in NS5A at baseline (OR, 7.78; $P<0.001)$, and other phase II and III trials also showed relatively low SVR12 with NS5A RAS (91\% compared with $99 \%$ without NS5A RAS). ${ }^{19,20}$ In this study, accurate diagnosis of cirrhosis using tissue biopsy or sequential LSM was limited, and analysis for NS5A RAS was not available. Instead, we used non-invasive fibrosis indices, such as APRI and FIB-4, which showed significant association with EOT and SVR12 in multivariate analysis. This result may be considered to be related to the maintenance of treatment for a relatively long period of time in patients with cirrhosis or patients who have failed treatment in the existing guidelines, and might be interpreted as using baseline or on-treatment non-invasive fibrosis markers that also can predict the failure of LDV/SOF treatment. In addition, the viral load of patients who failed to achieve ETR and SVR decreased during treatment, but increased again after treatment. This may be interpreted as that additional doses or another DAAs should be considered for patients with poor response after 12 weeks of treatment.

Serious AE associated with LDV/SOF was not observed. The AE profiles in this study were not different from those in previous clinical trials. The most frequent AE was headache followed by fatigue, and there was no $A E$ that resulted in dose interruption or treatment discontinuation. Consequently, LDV/SOF or LDV/SOF+ ribavirin may be safe for genotype $1 \mathrm{HCV}$-infected patients in real practice.

However, there are several limitations in this study. Firstly, LDV/ SOF is not a novel DAA treatment and is already substituted with pangenotypic DAAs. Although LDV/SOF treatment is not a novel issue, this large-scale cohort study was aimed to show the efficacy and safety of an affordable DAA for developing countries such as Mongolia. This study could be a good example of successful hepatitis $C$ treatment practice in developing countries where healthcare budgets are limited. Secondly, serum markers indicating tumor markers and renal functions were not analyzed. Thirdly, long-term SVR and its effect were not analyzed.

In conclusion, our data showed that LDV/SOF ( \pm ribavirin) regimen for 12 weeks was highly effective and safe for genotype 1
HCV-infected patients in the real-world setting, especially in patients with genotype $1 \mathrm{~b}$. Such results might be translated into other patients infected with HCV genotype 1 living in other AsianPacific area as a part of worldwide HCV eradication.

\section{Authors' contribution}

0 Baatarkhuu, JS Lee and J Amarsanaa: study concept and design, data acquisition, analysis, and interpretation; statistical analysis and manuscript drafting. DY Kim and SH Ahn: study concept and design, data analysis, and interpretation; manuscript drafting and critical revision; and study supervision. N Naranzul, D Enkhtuya, N Choijamts, P Batbayar, R Otgonbayar, BU Saruul, C Gantuul, B Gegeebadrakh, N Tuvshinbayar, D Badamsuren, G Ulzmaa, J Otgonbold and KH Han: data acquisition and critical manuscript revision.

\section{Acknowledgements}

We greatly appreciate the cooperation of the Ministry of Health of Mongolia, University General Hospital of the Mongolian National University of Medical Sciences, Mongolian Academy of Medical Sciences, Happy Veritas Liver Diagnostics Center, National Center for Communicable Diseases, Second State Central Hospital, Third State Central Hospital and the Mongolian Association for the Study of Liver Diseases for their provision of field staff.

We would like to express our special appreciation to Yonsei University College of Medicine of Korea for their tremendous efforts in the presenting study.

\section{Conflicts of Interest}

The authors have no conflicts to disclose.

\section{SUPPLEMENTARY MATERIAL}

Supplementary material is available at Clinical and Molecular Hepatology website (http://www.e-cmh.org).

\section{REFERENCES}

1. Baatarkhuu O, Kim DY, Ahn SH, Nymadawa P, Dahgwahdorj Y, Shagdarsuren $\mathrm{M}$, et al. Prevalence and genotype distribution of hepatitis C virus among apparently healthy individuals in Mongolia: a population-based nationwide study. Liver Int 2008;28:1389-1395.

2. Baatarkhuu O, Gerelchimeg T, Munkh-Orshikh D, Batsukh B, Saran- 
gua $G$, Amarsanaa J. Epidemiology, genotype distribution, prognosis, control, and management of viral hepatitis B, C, D, and hepatocellular carcinoma in Mongolia. Euroasian J Hepatogastroenterol 2018;8:57-62.

3. Baatarkhuu O, Uugantsetseg G, Munkh-Orshikh D, Naranzul N, Badamjav S, Tserendagva D, et al. Viral hepatitis and liver diseases in Mongolia. Euroasian J Hepatogastroenterol 2017;7:68-72.

4. Poordad F, McCone J Jr, Bacon BR, Bruno S, Manns MP, Sulkowski MS, et al. Boceprevir for untreated chronic HCV genotype 1 infection. N Engl J Med 2011;364:1195-1206.

5. Afdhal N, Zeuzem S, Kwo P, Chojkier M, Gitlin N, Puoti M, et al. Ledipasvir and sofosbuvir for untreated HCV genotype 1 infection. N Engl J Med 2014;370:1889-1898.

6. Afdhal N, Reddy KR, Nelson DR, Lawitz E, Gordon SC, Schiff E, et al. Ledipasvir and sofosbuvir for previously treated HCV genotype 1 infection. N Engl J Med 2014;370:1483-1493.

7. Zeng QL, Xu GH, Zhang JY, Li W, Zhang DW, Li ZQ, et al. Generic ledipasvir-sofosbuvir for patients with chronic hepatitis C: a real-life observational study. J Hepatol 2017;66:1123-1129.

8. Chon YE, Jung ES, Park JY, Kim DY, Ahn SH, Han KH, et al. The accuracy of noninvasive methods in predicting the development of hepatocellular carcinoma and hepatic decompensation in patients with chronic hepatitis B. J Clin Gastroenterol 2012;46:518-525.

9. Lee HW, Chon YE, Kim SU, Kim BK, Park JY, Kim DY, et al. Predicting liver-related events using transient elastography in chronic hepatitis c patients with sustained virological response. Gut Liver 2016;10:429-436.

10. Jung KS, Kim SU. Clinical applications of transient elastography. Clin Mol Hepatol 2012;18:163-173.

11. Sterling RK, Lissen E, Clumeck N, Sola R, Correa MC, Montaner J, et al. Development of a simple noninvasive index to predict significant fibrosis in patients with HIV/HCV coinfection. Hepatology 2006;43:1317-1325.
12. Lai CL, Wong VW, Yuen MF, Yang JC, Knox SJ, Mo H, et al. Sofosbuvir plus ribavirin for the treatment of patients with chronic genotype 1 or 6 hepatitis C virus infection in Hong Kong. Aliment Pharmacol Ther 2016:43:96-101.

13. Wai CT, Greenson JK, Fontana RJ, Kalbfleisch JD, Marrero JA, Conjeevaram HS, et al. A simple noninvasive index can predict both significant fibrosis and cirrhosis in patients with chronic hepatitis $C$. Hepatology 2003;38:518-526.

14. Charlton M, Everson GT, Flamm SL, Kumar P, Landis C, Brown RS Jr, et al. Ledipasvir and sofosbuvir plus ribavirin for treatment of HCV infection in patients with advanced liver disease. Gastroenterology 2015;149:649-659.

15. European Association for the Study of the Liver. EASL recommendations on treatment of hepatitis C 2018. J Hepatol 2018;69:461-511.

16. Omata $M$, Kanda $T$, Wei L, Yu ML, Chuang WL, Ibrahim A, et al. APASL consensus statements and recommendation on treatment of hepatitis C. Hepatol Int 2016;10:702-726.

17. AASLD-IDSA HCV Guidance Panel. Hepatitis C guidance 2018 update: AASLD-IDSA recommendations for testing, managing, and treating hepatitis C virus infection. Clin Infect Dis 2018;67:14771492.

18. European Association for the Study of the Liver. EASL recommendations on treatment of hepatitis C 2016. J Hepatol 2017;66:153-194.

19. Tsuji K, Kurosaki M, Itakura J, Mori N, Takaki S, Hasebe C, et al. Real-world efficacy and safety of ledipasvir and sofosbuvir in patients with hepatitis C virus genotype 1 infection: a nationwide multicenter study by the Japanese Red Cross Liver Study Group. J Gastroenterol 2018;53:1142-1150.

20. Zeuzem S, Mizokami M, Pianko S, Mangia A, Han KH, Martin R, et al. NS5A resistance-associated substitutions in patients with genotype 1 hepatitis C virus: prevalence and effect on treatment outcome. J Hepatol 2017;66:910-918. 